\title{
The $t$-pebbling number is eventually linear in $t$
}

\author{
Michael Hoffmann \\ Jiř́i Matoušek \\ ETH Zürich, Switzerland \\ Charles University, Prague, Czech Republic \\ hoffmann@inf .ethz.ch \\ matousek@kam.mff.cuni.cz \\ Yoshio Okamoto* \\ Philipp Zumstein \\ JAIST, Nomi, Japan \\ ETH Zürich, Switzerland \\ okamotoy@jaist.ac.jp \\ zuphilip@inf .ethz.ch
}

Submitted: Apr 4, 2010; Accepted: Jun 22, 2011; Published: Jul 22, 2011

Mathematics Subject Classification: 05C99, 05C35

\begin{abstract}
In graph pebbling games, one considers a distribution of pebbles on the vertices of a graph, and a pebbling move consists of taking two pebbles off one vertex and placing one on an adjacent vertex. The $t$-pebbling number $\pi_{t}(G)$ of a graph $G$ is the smallest $m$ such that for every initial distribution of $m$ pebbles on $V(G)$ and every target vertex $x$ there exists a sequence of pebbling moves leading to a distibution with at least $t$ pebbles at $x$. Answering a question of Sieben, we show that for every graph $G, \pi_{t}(G)$ is eventually linear in $t$; that is, there are numbers $a, b, t_{0}$ such that $\pi_{t}(G)=a t+b$ for all $t \geq t_{0}$. Our result is also valid for weighted graphs, where every edge $e=\{u, v\}$ has some integer weight $\omega(e) \geq 2$, and a pebbling move from $u$ to $v$ removes $\omega(e)$ pebbles at $u$ and adds one pebble to $v$.
\end{abstract}

\section{Introduction}

Let $G=(V, E)$ be an undirected graph. A pebbling distribution on $G$ is a function $p: V \rightarrow \mathbb{N}_{0}=\{0,1,2, \ldots\}$. A pebbling move consists of taking two pebbles off a vertex $u$ and adding one pebble on an adjacent vertex $v$ (we can think of this as paying a toll of one pebble for using the edge $\{u, v\}$ ). We also say that we move one pebble from $u$ to $v$.

More generally, we consider a graph $G$ together with a weight function $\omega: E \rightarrow$ $\{2,3,4, \ldots\}$ on edges. If an edge $e=\{u, v\}$ has weight $\omega(e)$, then we pay a toll of

\footnotetext{
*Supported by Global COE Program "Computationism as a Foundation for the Sciences" and Grantin-Aid for Scientific Research from Ministry of Education, Science and Culture, Japan, and Japan Society for the Promotion of Science.
} 
$\omega(e)-1$ pebbles for moving one pebble along $e$. (So the unweighted case corresponds to $\omega(v)=2$ for all $v \in V(G)$.)

More formally, if $e=\{u, v\} \in E$ and $p$ is a pebbling distribution such that $p(u) \geq \omega(e)$, then a pebbling move allows us to replace $p$ with the distriution $p^{\prime}$ given by

$$
p^{\prime}(w)= \begin{cases}p(u)-\omega(e) & \text { for } w=u \\ p(v)+1 & \text { for } w=v \\ p(w) & \text { otherwise }\end{cases}
$$

For a vertex $x \in V(G)$, let $\pi_{t}(G, \omega, x)$ be the smallest integer $m$ such for all distributions $p$ of $m$ pebbles there is a distribution $q$ with $q(x) \geq t$ that can be reached from $p$ by a sequence of pebbling moves. The t-pebbling number of $(G, \omega)$ is defined as $\pi_{t}(G, \omega)=\max \left\{\pi_{t}(G, \omega, x): x \in V(G)\right\}$ and we write $\pi_{t}(G)$ for the unweighted case with $\omega \equiv 2$.

Graph pebbling originated in combinatorial number theory and group theory. The pebbling game (unweighted and with $t=1$ ) was suggested by Lagarias and Saks, and in print it first appears in Chung [2]. For more background we refer to two recent surveys by Hurlbert $[4,5]$.

For some graph classes the (unweighted) $t$-pebbling number has been determined exactly. We have $\pi_{t}\left(K_{n}\right)=2 t+n-2$ for the complete graph, $\pi_{t}\left(C_{2 n}\right)=t 2^{n}$ and $\pi_{t}\left(C_{2 n-1}\right)=t 2^{n-1}+2\left\lfloor\frac{2^{n}}{3}\right\rfloor-2^{n-1}+1$ for the cycle, and $\pi_{t}\left(Q_{d}\right)=t 2^{d}$ for the cube (see [7]). All of these are linear functions of $t$. Moreover, one can show that the $t$-pebbling number of any tree is linear in $t$ by using the methods of [8]. It is shown in [6] that for the complete bipartite graph, we have $\pi_{t}\left(K_{m, n}\right)=\max \{2 t+m+n-2,4 t+m-2\}$, which is linear in $t$ but only for $t$ sufficiently large.

Sieben [8] asked whether the $t$-pebbling number is always linear for $t \geq t_{0}$ where $t_{0}$ is some constant. We answer this question affirmatively. A similar result is known in Ramsey theory: the Ramsey number of $t$ copies of a graph $\mathrm{G}$ is eventually linear in $t$ (see [1]).

To formulate our result, let us define, for every two vertices $u, v \in V(G)$, the multiplicative distance

$$
\operatorname{mdist}(u, v):=\min \left\{\prod_{e \in E(P)} \omega(e): P \text { is a } u \text {-v-path in } G\right\},
$$

(in particular, $\operatorname{mdist}(u, u)=1$ because the empty product equals 1 ). The function $\log (\operatorname{mdist}(u, v))$ clearly defines a metric on $V(G)$. Further, for $x \in V(G)$ we set

$$
r_{x}:=\max \{\operatorname{mdist}(x, v): v \in V(G)\} .
$$

Theorem 1. For every graph $G$ with edge weight function $\omega$ and for every $x \in V(G)$ there exist $b$ and $t_{0}$ such that that for all $t \geq t_{0}$

$$
\pi_{t}(G, \omega, x)=r_{x} t+b .
$$

Consequently, for a suitable $t_{0}=t_{0}(G, \omega), \pi_{t}(G, \omega)$ is a linear function of $t$ for all $t \geq t_{0}$. 
As a corollary, we immediately obtain a result from Hersovici et al. [3] about fractional pebbling:

$$
\lim _{t \rightarrow \infty} \frac{\pi_{t}(G)}{t}=2^{\operatorname{diam}(G)}
$$

where $\operatorname{diam}(G)$ denotes the diameter of $G$ in the usual shortest-path metric. Indeed, for the weight function $\omega \equiv 2$ we have $\max _{x \in V(G)} r_{x}=2^{\operatorname{diam}(G)}$.

Unfortunately, our proof of Theorem 1 is existential, and it yields no upper bound on $t_{0}$. It would be interesting to find upper bounds on (the minimum necessary) $t_{0}$ in terms of $G$ and $\omega$, or lower bounds showing that a large $t_{0}$ may sometimes be needed.

\section{Proof of Theorem 1}

First we check that

$$
\pi_{t}(G, \omega, x) \geq r_{x} t
$$

for all $t$. To this end, we consider the distribution $p_{0}$ with $r_{x} t-1$ pebbles, all placed at a vertex $y$ with $\operatorname{mdist}(x, y)=r_{x}$. We claim that, starting with $p_{0}$, it is impossible to obtain $t$ pebbles at $x$.

To check this, we define the potential of a pebbling distribution $p$ as

$$
F(p):=\sum_{v \in V(G)} \frac{p(v)}{\operatorname{mdist}(v, x)} .
$$

It is easy to see that this potential is nonincreasing under pebbling moves (using the "multiplicative triangle inequality" $\operatorname{mdist}(u, x) \leq \omega(\{u, v\}) \operatorname{mdist}(v, x))$. Now $F\left(p_{0}\right)<t$, while any distribution $q$ with at least $t$ pebbles at $x$ has $F(q) \geq t$, which proves the claim and thus also (1).

Next, we define the function

$$
f(t):=\pi_{t}(G, \omega, x)-r_{x} t .
$$

We have $f(t) \geq 0$ for all $t$ by $(1)$. Let $n:=|V(G)|$; we claim that $f$ is nonincreasing for all $t \geq n$. Once we show this, Theorem 1 will be proved, since a nonincreasing nonnegative function with integer values has to be eventually constant.

So we want to prove that, for all $t \geq n$, we have $f(t) \leq f(t-1)$, which we rewrite to

$$
\pi_{t}(G, \omega, x) \leq \pi_{t-1}(G, \omega, x)+r_{x} .
$$

To this end, we consider an arbitrary pebbling distribution $p$ with $m:=\pi_{t-1}(G, \omega, x)+$ $r_{x}$ pebbles. By (1) we obtain $m \geq r_{x}(t-1)+r_{x}=r_{x} t \geq r_{x} n$. So by the pigeonhole principle, there exists a vertex $y$ with $p(y) \geq r_{x}$.

Let us temporarily remove $r_{x}$ pebbles from $y$. This yields a distribution with at least $\pi_{t-1}(G, \omega, x)$ pebbles, and, by definition, we can convert it by pebbling moves to a distribution with at least $t-1$ pebbles at $x$. Now we add the $r_{x}$ pebbles back to $y$ and move them toward $x$, and in this way we obtain one additional pebble at $x$. This verifies (2), and the proof of Theorem 1 is finished. 


\section{References}

[1] S. Burr, P. Erdős, J. Spencer. Ramsey theorems for multiple copies of graphs. Trans. of the American Mathematical Society. 209 (1975) 87-99.

[2] F. R. K. Chung. Pebbling in hypercubes. SIAM J. Discrete Math. 2 (1989) 467-472.

[3] D. S. Hersovici, B. D. Hester, G. H. Hurlbert. Diameter bounds, fractional pebbling, and pebbling with arbitrary target distributions. ArXiv preprint, http://arxiv . org/abs/0905.3949.

[4] G. Hurlbert. A survey of graph pebbling. Congressus Numerantium 139 (1999) 41-64.

[5] G. Hurlbert. Recent progress in graph pebbling. Graph Theory Notes of New York XLIX (2005) 25-37.

[6] A. Lourdusamy, A. Punitha. On t-pebbling graphs. Utilitas Math., to appear, 2010.

[7] A. Lourdusamy and S. Somasundaram. The $t$-pebbling number of graphs. Southeast Asian Bull. Math. 30 (2006) 907-914.

[8] N. Sieben. A graph pebbling algorithm on weighted graphs. J. of Graph Algorithms and Applications. 14 (2010) 221-244. 